\title{
BPM System for the SNS Ring and Transfer Lines ${ }^{1}$
}

\author{
W. C. Dawson, P. Cameron, P. Cerniglia, J. Cupolo, C. Degen, A. \\ DellaPenna, A. Huhn, M. Kesselman, J. Mead, R. Sikora \\ Brookhaven National Laboratory, Upton, NY 11973, USA
}

\begin{abstract}
The Spallation Neutron Source Ring accumulates about 1060 pulses of $38 \mathrm{~mA}$ peak current $1 \mathrm{GeV} \mathrm{H}$-minus particles from the Linac thru the HEBT line, then delivers this accumulated beam in a single pulse to the mercury target via the RTBT line. Bunching frequency of beam in the HEBT line is $402.5 \mathrm{MHz}$, and about $1 \mathrm{MHz}$ in the Ring and RTBT. Position monitor electrodes in HEBT are of the shorted stripline type, with apertures of $12 \mathrm{~cm}$ except in the dispersive bend, where the aperture is $21 \mathrm{~cm}$. Ring and RTBT electrodes are open striplines, with apertures of $21,26,30$, and $36 \mathrm{~cm}$. All pickups are dual plane. The electronics will be PC-based with the Analog/Digital Front End passing data and receiving control and timing thru a custom PCI interface developed by LANL[1]. LabVIEW will be used to direct the acquisition, process the data, and transfer results via Ethernet to the EPICS control system. To handle the dynamic range required with well over $60 \mathrm{~dB}$ variation in signal size, the Ring and RTBT electronics will employ a fast gain switching technique that will take advantage of the $300 \mathrm{~ns}$ tail-to-head gap to provide position measurement during the entire accumulation cycle. Beam-based alignment will be utilized as part of the system calibration.
\end{abstract}

\section{PERTINENT HEBT-Ring- RTBT REQUIREMENTS}

1- Intensity

2- Pulse length

3- Accuracy

4- Resolution

5- Turn by turn data desired
$5 \mathrm{E} 10-2 \mathrm{E} 14$ protons per pulse

$.3-1000 \mu \mathrm{Sec}$

$+/-1 \mathrm{~mm}$

$0.15 \mathrm{~mm}$

\footnotetext{
${ }^{1}$ Work performed under the auspices of the U.S. Department of Energy
} 


\section{CHALLENGES}

1- Single turn (commissioning) vs. multi-turn operation

2- Dynamic Range

3- Resolution and system band-width selection

4- Cable reflections due to out-of-band mismatch

5- Method of position calculation

6- Linearity over a large range of aperture

7- Beam-based alignment

8- Possible use of the PUE's as electron clearing electrodes

9- Monitoring a tune measurement stimulus signal at $\sim 50 \mathrm{MHz}$

\section{GENERAL DISCUSSION}

The beam in the HEBT is primarily composed of short microbunches. These give rise to a large $\mathrm{RF}$ component at the $402.5 \mathrm{MHz}$ bunching frequency and it's harmonics. The character of the pick-up signals in this region of the SNS is the same as that of the Linac section, and it is intended to use identical electronics to that of the Linac in this region. The Ring accumulates the injected bunches from the HEBT, and after a number of turns the charge diffuses and the RF character is quickly lost. With a millisecond of injection and about a 1. MHz revolution frequency, the charge will grow by a factor of about 1000 . Early injected turns will have RF structure, while turns that have been accumulated for many turns will have the character of a $645 \mathrm{~ns}$ pulse with a repetition rate of $945 \mathrm{~ns}$. The pulses with little RF structure will provide a base-band signal, while those that have recently been injected will provide an RF character. Therefore, to obtain position information two types of electronics are required. For early turns something similar to the Linac electronics is necessary, while for the later turns a base-band approach is required. Position monitoring in the SNS Ring has been addressed in two previous publications $[2,3]$.

The RTBT has a beam that displays primarily the character of a single bunch of particles for $645 \mathrm{~ns}$. Therefore, this is similar to the Ring base-band signal, and electronics based upon this design would be most appropriate. During commissioning and studies, however, one could expect to see a single mini-pulse of microbunches transported through the entire system. To observe this, electronics similar to that used in the Linac are required.

To measure the tune, the plan is to excite the beam with a stimulus signal in the range of 40 to $60 \mathrm{MHz}$ and observe the response with a resonant pickup. Modification of the input filtering and mixing scheme to accommodate this measurement is being considered. 
Electron cloud production is also expected to be a problem in the SNS. The use of the 44 Ring BPM pickup electrodes as clearing electrodes is being considered. This will involve applying a potential difference of $2 \mathrm{KV}$ to the electrodes to sweep out electrons that originate at the walls during the later portion of the $645 \mathrm{~ns}$ bunch. This DC signal will be applied through the signal cables and must be isolated from the input to the electronics (Ring BPMs are of the open stripline design).

A bcam based alignment technique will be employed in the Ring to establish the location of the BPM electrical center relative to the adjacent quadrupole magnetic center. A processing scheme employing normalization will eliminate gain from the results, and the calculated position will become dependent upon the basic sensitivity of the BPM.

\section{BACKGROUND}

This system is presently in the preliminary design stage. The original thought was to utilize a number of Linac BPM electronic systems [1] to get a general handle on beam position for early turns. For position measurement through the accumulation cycle the basc-band concept was pursued and a basic design scheme was developed. This included a switched gain concept that follows that used in the beam current monitor system [4]. The basic concept provides different paths for the signal to follow, each with different gain. The paths are summed in a sum stage. Each path is capable of being switched, in or out, by a logic signal to a switched amplifier (OPA3680). In so doing, the gain can be switched within $100 \mathrm{~ns}$, sufficient for switching during the beam "gap" time in the Ring.

To permit a simpler and more general solution to commissioning the Ring and in the interest of project-wide commonality, an approach that utilizes the electronics of the Linac is under consideration, with some modifications to permit it's use with baseband signals. This basic concept uses the RF sensitive electronics for early turns and during the commissioning process, while allowing for switching the mixer off, and providing base-band filtering only, to permit the base-band signal to propagate through the system to the ADC. Processing will continue in the normal manner, and the data will be analyzed for position. The Ring and RTBT do not require the phase information, and the I/Q demodulation present in the Linac electronics need not be used. In addition, it is possible that some of the calibration-switching circuitry may be replaced by local oscillator switches and front end filters to conserve circuit board real estate. There will be some calibration switching necessary to characterize each gain path of each channel.

A digital front end (DFE) board and PCI interface board similar to those used in the linac design will be used to digitize the signals, and allow transfer of information to and from a PC. LabVIEWTM will perform control, signal processing and analysis as well as final communication, through Ethernet, with the control system. 


\section{DETAILS}

The basic design centers upon the Bergoz ${ }^{\circledR}$ BPM Analog Front End (AFE), designed for the Linac. A simplificd block diagram is shown in Figure 1. This diagram shows an example of a $402.5 \mathrm{MHz}$ AFE. The pickup signals are passed through a switching matrix that permits calibration information to be placed on the inputs to the electronics or the BPM pickup elements. These signals are band pass filtered to allow only the $402.5 \mathrm{MHz}$ information to pass. They are amplified as necessary, and mixed with a local oscillator of $352.5 \mathrm{MHz}$. This yields a difference frequency of $50 \mathrm{MHz}$, which is selected by another band pass filter. The result is digitized by a $40 \mathrm{MHz}$ digitizer. This digitizer brings the resulting signal to base-band, and the information of interest is a $5 \mathrm{MHz}$ band after I/Q demodulation of the $10 \mathrm{MHz}$ down-converted signal.

The modified design would eliminate some of the calibration switching, since the Ring will be calibrated by beam-based alignment. Some switching will be required to establish filtering and channel gain. The initial band-pass filtering will be switch selected to be either at RF or a $5 \mathrm{MHz}$ low pass. For base-band operation the mixer will be biased to avoid mixing. The $50 \mathrm{MHz}$ filter already designed for this application can be removed for the base-band application.

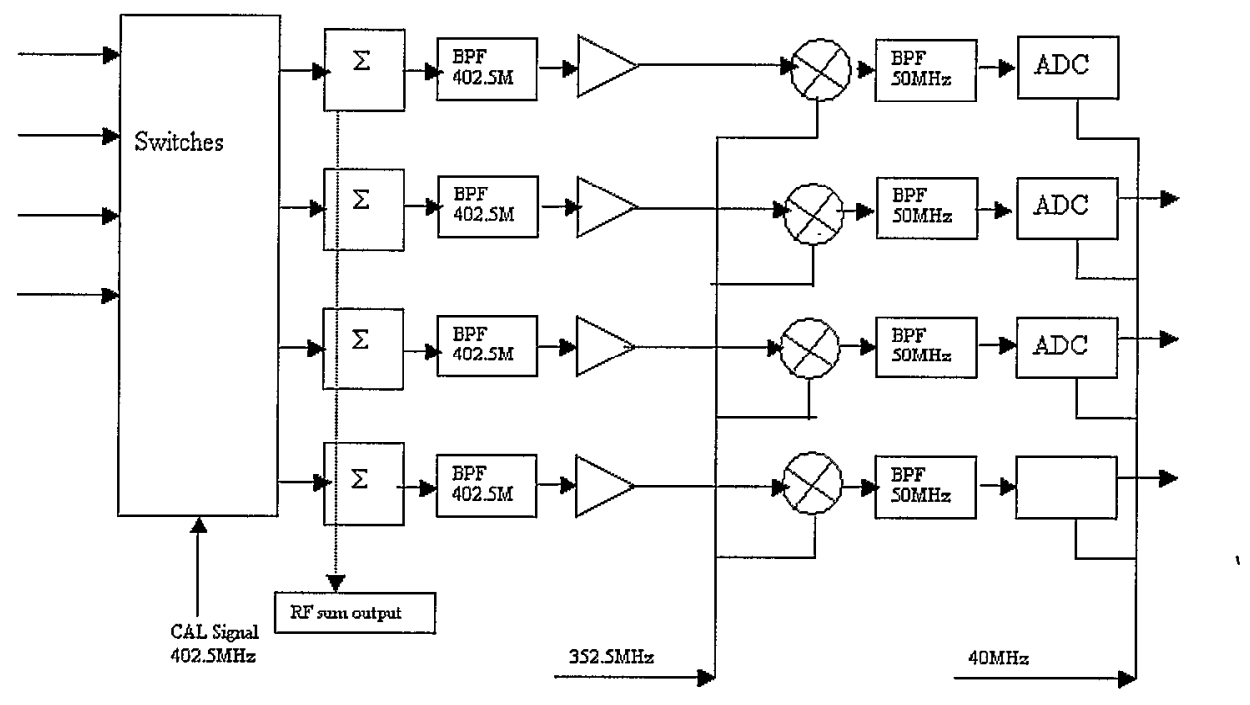

FIGURE 1. Block diagram of basic Linac BPM Electronics 
Since I/Q demodulation is not required (not interested in phase in the Ring and RTBT), the available ADC bandwidth is $20 \mathrm{MHz}$ when clocked at $40 \mathrm{MHz}$. The required bandwidth is a trade-off between settling time in less than 300ns, noise considerations, signal sensitivity, and signal level at high current. A bandwidth of $5 \mathrm{MHz}$ is the preliminary selection to meet these considerations.

In addition to the dual operating modes mentioned above, it would also be desirable to examine the spectral lines of the tune system. This could be accommodated with another modification, now under investigation, that allowed a spectrum of $40 \mathrm{MHz}$ to $60 \mathrm{MHz}$ to be processed. If this was delivered to the $A D C$, and sampled at $40 \mathrm{MHz}$, the spectrum would be brought back to base-band and occupy a $20 \mathrm{MHz}$ bandwidth. This could be analyzed by an FFT algorithm to obtain the spectrum.

\section{Dynamic Range and Bandwidth}

One of the design challenges is to deal with a very large dynamic range in the Ring and RTBT. The signal is expected to change by a factor of about 1000 due to the storage of 1060 turns. In addition the requirement of $0.15 \mathrm{~mm}$ resolution will necessitate an additional 1000 for a total of 6 orders of magnitude. It would be nice to adjust gain in such a way that we lose no turn information. Input signal voltages could be quite large directly out of the BPM (very wide-band, $20 \mathrm{mV}$ to $200 \mathrm{~V}$ peak). When filtered to $5 \mathrm{MHz}$, signals will range from $1.8 \mathrm{mV}$ peak to 10 Volts peak, for $15 \mathrm{ma}$ and $100 \mathrm{amp}$ beams with no allowances for terminations and matching circuits. (When filtered to $10 \mathrm{MHz}$ signals will range from $3.2 \mathrm{mV}$ to $15.3 \mathrm{~V}$.) An accuracy of $1 \mathrm{~mm}$ and a resolution of better than $0.15 \mathrm{~mm}$ is the present requirement. To achieve this a signal to noise ratio of $48 \mathrm{~dB}$ is required [5]. This is $3.2 \mathrm{nV} / \mathrm{rtHz}$ for a $5 \mathrm{MHz}$ bandwidth. A 50 Ohm resistor $(0.91 \mathrm{nV} / \mathrm{rtHz})$ and a typical $2.4 \mathrm{nV} / \mathrm{rtHz}$ amplifier will yield $2.57 \mathrm{nV} / \mathrm{rtHz}$, allowing for little additional resistor noise and signal attenuation due to cabling and "real" filters. A single bunch with RF structure will provide near a $100 \mathrm{~dB}$ signal to noise ratio. Therefore, for the single turn study case, using the $\mathrm{RF}$ detection scheme of the Linac is suggested. A single full current injected turn will yield a signal of about $4.5 \mathrm{mV}(38 \mathrm{~mA})$. This is $8 \mathrm{db}$ more signal than the study current, providing the much needed additional signal to meet the resolution requirement. If the mixer switching speed permits, additional noise margin can be achieved by selecting baseband operation at turns 2 or 3 . The system bandwidth clearly affects the noise and in-turn the resolution. The wider bandwidth improves signal almost proportionally, due to the high harmonic content in the signal, while increasing noise by the square root. However, the higher signals need to be dealt with by protecting the amplifiers carefully at the other end of the signal envelope. A compromise to $5 \mathrm{MHz}$ was established. 


\section{Cable Reflections}

A good match at the input is required to assure minimal reflection back to the BPM PUE. In addition to the undesired beam kick which would result, the striplines are unterminated and will reflect signal back yet again. This could interfere with data from the next revolution for cables $100 \mathrm{~m}$ to $200 \mathrm{~m}$ long. Three methods have been investigated to match the cable over a wide band; a diplexer, an attenuator, or a combination of both. A simple two-pole diplexer can be constructed using a resistively terminated inductor and capacitor in a low-pass configuration, in parallel with a resistively terminated capacitor and inductor in a high-pass configuration. If the $\mathrm{L} / \mathrm{C}$ ratio is 2 times the square of the load impedance, such a configuration will have constant input impedance. Difficulties arise using real components, and a better match may be achievable by using a simple resistive attenuator at the expense of signal. If enough signal is available one could use both.

\section{Signal Processing}

The standard difference over sum or log ratio method of processing the data will provide information weighted near the beam edges for signals sampled near their peak. To obtain a position more indicative of the average position over the entire mini-pulse a mean square approach is under investigation. With the beam near the center, signals on opposite elements are proportional to the current (I) times (1+ar) for one element, and (1-ar) for the opposite element. The constant a is related to the BPM dimensions, and $\mathrm{r}$ is the radial beam position along the axis of the elements. Therefore, the difference over the sum yields a sensitivity of approximately $2 \mathrm{ar} / 2 \cong$ ar for $\mathrm{ar}<<1$. Processing the data as mean squared power instead of a peak signal measurement can double the sensitivity of the BPM. That is; $\left[(1+a r)^{2}-(1-a r)^{2}\right] /\left[(1+a r)^{2}+(1-a r)^{2}\right] \cong$ $4 \mathrm{ar} / 2=2 \mathrm{ar}$ for $\mathrm{ar}<<1$.

To a first order approximation, gain-error affects only a DC error. When employing beam-based alignment the DC term is eliminated, leaving residual second order affect to the linearity with powers of $r$. In addition, since these signals are averaged over an entire mini-pulse $(\sim 1 \mu \mathrm{s})$, or a number of mini-pulses, noise term contributions can be reduced. Some of the noise terms are estimates of the noise statistics that do not change with time, and can be characterized for partial reduction, while other noise terms involve the average of the product of noise with signal. This average tends to zero as the averaging time is increased. With increased sensitivity and noise reduction, an improvement in resolution over the standard difference over sum approach is expected. Work continues along these lines to better understand the process. 


\section{Linearity}

The linearity has been shown to be improved by using $\log$ ratio processing. By doing the log ratio analysis of the mean squared power, calculated similarly to that indicated above, the BPM response can be made to be more linear over a larger range of the pipe radius. This calculation need be made only once per position analysis (after calculating a right and left mean square with noise subtraction).

\section{Beam-based Alignment}

The location of the HEBT, Ring, and RTBT BPM PUEs relative to the magnetic centers of the adjacent quadrupoles will be determined by beam-based alignment[6,7]. Trim windings with $30 \mathrm{~A}$ current capacity are available on all quadrupoles, resulting in a $2 \%$ modulation of field strength. These winding can be driven with a frequency of about $10 \mathrm{~Hz}$. Beam which passes off-center through a modulated quadrupole will experience a transverse kick. This position oscillation can be detected by any BPM of the proper phase relative to the kick. One possibility is to use the acquisition system of the tune meter [8] to detect this motion. If the beam is excited by the $\sim 50 \mathrm{MHz}$ tune kicker simultaneous with the quadrupole modulation, this modulation will appear as $\sim 10 \mathrm{~Hz}$ sidebands on the tune signal. This approach benefits both from the sensitivity of the resonant pickups used for the tune system and the signal enhancement of the carrier which results from driving the beam on resonance with the tune system. During SNS commissioning dedicated time will be required to accomplish the initial measurement of the Quadrupole-BPM offset. During normal operations beam-based alignment can also be accomplished continuously and parasitically by taking data over many accumulation cycles if sufficient care is given to maintaining phase coherence between cycles. It is estimated that the precision of the determined offsets should be about 100 microns.

\section{SUMMARY}

A description of the SNS Ring BPM electronics, presently in the preliminary design phase, has been presented. The proposed system incorporates a single versatile electronics scheme to accommodate both signals with RF structure and signals with primarily base-band structure. This will permit sensitivity for single turn studies as well as accumulated stored beam operation. A protected switched gain path scheme, similar to the SNS BCM system will provide gain changes to handle the large dynamic range. In addition, the electronics will be made flexible to permit other uses for the BPM signals (tune measurement diagnostic). To make the position calculation more indicative of the average beam position over an entire mini-pulse a mean square approach to data processing is under investigation. The system will be aligned using 
beam-based alignment. The Ring BPMs are of the open stripline design and may be used as electron clearing electrodes in the future.

\section{ACKNOWLEDGMENTS}

The authors would like to acknowledge Tom Shea of ORNL for his assistance with the basic concepts.

\section{REFERENCES}

1. J. Power et al, "Beam Position Monitors for the SNS Linac", PAC2001, NY.

2. J. Beebe-Wang et-al, "Simulations of SNS Accumulator Ring Beam Position Monitor Signals", BNL/SNS Tech Note 38, November 1997.

3. T.J. Shea et-al, "Design Study of Position Monitoring in the SNS Ring", BNL SNS Tech Note (unpublished).

4. M. Kesselman et-al, "Spallation Neutron Source Beam Current Monitor Electronics", these proceedings.

5. R. Shafer, "Beam Position Monitoring"; AIP Conference Proceedings 212, p 46 (1989).

6. B. Dehning et-al, "Dynamic Beam-Based Calibration of Beam Position Monitors", CERN SL-98-038 BI.

7. P. Tcncnbaum and T. Raubcnhcimcr, "Resolution and systematic limitations in beam-based alignment", Phys Rev Special Topics - Accelerators and Beams, V3, 052801 (2000).

8. P. Cameron et al, "Tune Measurement in the SNS Ring", these proceedings. 